\title{
Cerebral Salt Wasting Syndrome and Systemic Lupus Erythematosus: Case Report
}

\author{
Filipe Martins ${ }^{\mathrm{a}, \mathrm{c}}$, Carolina Ourique ${ }^{\mathrm{a}}$, Jose Faria da Costa ${ }^{\mathrm{a}}$, Joao Nuak ${ }^{\mathrm{b}}$, Vitor Braz ${ }^{\mathrm{a}}$, \\ Edite Pereira $^{\mathrm{a}}$, Antonio Sarmento ${ }^{\mathrm{b}}$, Jorge Almeida ${ }^{\mathrm{a}}$
}

\begin{abstract}
Cerebral salt wasting (CSW) is a rare cause of hypoosmolar hyponatremia usually associated with acute intracranial disease characterized by extracellular volume depletion due to inappropriate sodium wasting in the urine. We report a case of a 46-year-old male with recently diagnosed systemic lupus erythematosus (SLE) initially presenting with neurological involvement and an antiphospholipid syndrome (APS) who was admitted because of chronic asymptomatic hyponatremia previously assumed as secondary to syndrome of inappropriate antidiuretic hormone secretion (SIADH). Initial evaluation revealed a hypoosmolar hyponatremia with high urine osmolality and elevated urinary sodium concentration. Clinically, the patient's extracellular volume status was difficult to define accurately. After exclusion of other etiologies, a diagnosis of CSW was established and the patient started appropriate treatment with normalization of sodium levels. The challenge in diagnosing CSW relies on the differentiation from the SIADH, since it shares most of the laboratory features. The critical difference is the state of extracellular volume which can be difficult to access accurately in routine clinical practice. There is evidence that the fractional excretion of urate (FEUrate) can be of assistance. The relationship between SLE and hyponatremia is mostly limited to adverse pharmacological side effects, so to our knowledge, this is the first association between SLE with neurological involvement and CSW.
\end{abstract}

Keywords: Cerebral salt wasting syndrome; Hyponatremia; Neurolupus; Systemic lupus erythematosus

\section{Introduction}

The term cerebral salt wasting (CSW) refers to a renal loss of sodium, originally thought to be exclusive of intracranial

\footnotetext{
Manuscript accepted for publication August 12, 2016

anternal Medicine Department, Centro Hospitalar Sao Joao, Porto, Portugal ' Infectious Diseases Department, Centro Hospitalar Sao Joao, Porto, Portugal ${ }^{\mathrm{c} C}$ Corresponding Author: Filipe Martins, Internal Medicine Department, Centro Hospitalar Sao Joao, Alameda Professor Hernani Monteiro, 4200 Porto, Portugal.Email: futins@gmail.com
}

doi: http://dx.doi.org/10.14740/jmc2615w disorders, that results in hyponatremia and a decrease in extracellular fluid volume. It is characterized by a hypotonic hyponatremia with inappropriately elevated urine sodium concentration in the setting of a normal kidney function [1-3].

The onset of this disorder is typically seen within the first 10 days following a neurological insult and usually lasts no more than 1 week [1, 2]. Pathophysiology is not completely understood but the major mechanism might be the inappropriate and excessive release of natriuretic peptides which would result in natriuresis and volume depletion. A secondary neurohormonal response would result in an increase in the reninangiotensin system and consequently in antidiuretic hormone $(\mathrm{ADH})$ production. Since the volume stimulus is more potent than the osmolar stimulus, the ADH secretion overrides the usual inhibition by the coexisting plasma hypoosmolality, thus perpetuating the hyponatremia [2-5].

The main differential diagnosis is the syndrome of inappropriate antidiuretic hormone secretion (SIADH) which shares most of the laboratory findings, making a definitive diagnosis challenging. The only clinical difference is the state of the extracellular volume, being euvolemic or hypervolemic in SIADH and hypovolemic in renal salt wasting (RSW) [4, $6,7]$. This is important because treatment strategies are very different.

We report a case of a male patient with recently diagnosed systemic lupus erythematous (SLE) with central nervous system (CNS) involvement with CSW. To our knowledge, this is the first documented case in the literature.

\section{Case Report}

We report a case of a 46-year-old male with a past history relevant for autoimmune thyroiditis with hypothyroidism (medicated with levothyroxine $0.075 \mathrm{mg}$ i.d.), SLE with severe CNS involvement (diagnosed 9 months before in the setting of a coma of unknown origin, treated with pulses of cyclophosphamide and methylprednisolone, currently with prednisolone 20 $\mathrm{mg}$ i.d. and azathioprine $50 \mathrm{mg}$ i.d.), and antiphospholipid syndrome (ischemic cerebral lesions and previous venous thromboembolism with positivity for anticardiolipin $\operatorname{IgG}$ ), who was admitted because of refractory asymptomatic hyponatremia $(120-125 \mathrm{mEq} / \mathrm{L})$ previously interpreted as secondary to SI$\mathrm{ADH}$, possibly due to the cyclophosphamide pulses, and managed with fluid restriction. The evidence of the dysnatremia dated from the previous hospital stay when the neurolupus was 


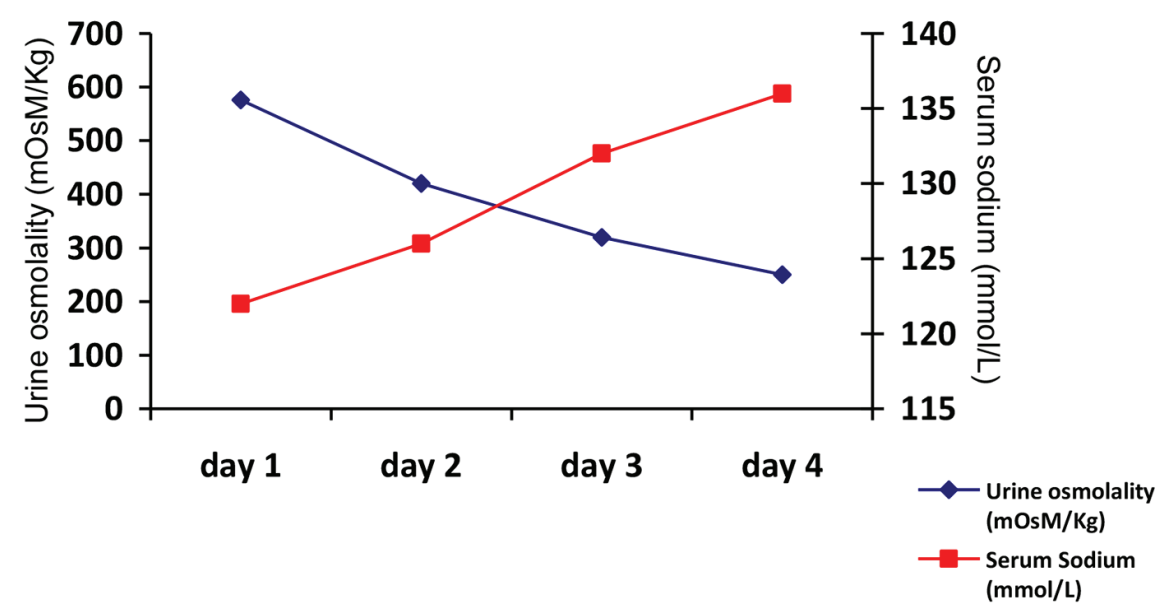

Figure 1. Urine osmolality and serum sodium concentration evolution during saline infusion.

diagnosed.

On physical examination, the patient was alert, responsive, asymptomatic, and apparently hydrated; blood pressure was $110 / 66 \mathrm{~mm} \mathrm{Hg}$, and pulse was 88 beats per minute. Cardiopulmonary examination was unremarkable. Abdominal examination was painless with no masses and no peripheral edema. Lab workup showed no anemia with hemoglobin $12.1 \mathrm{~g} / \mathrm{dL}$, hematocrit $36.1 \%$, normal white blood count, mild thrombocytopenia $110 \times 10^{12} / \mathrm{L}$, blood urea $70 \mathrm{mg} / \mathrm{dL}$, blood creatinine $1.05 \mathrm{mg} / \mathrm{dL}$, uric acid $4 \mathrm{mg} / \mathrm{dL}$, hyponatremia $122 \mathrm{mEq} / \mathrm{L}$ with no other ionic abnormalities, serum osmolality $260 \mathrm{mOsm} / \mathrm{kg}$, urinary osmolality $420 \mathrm{mOsm} / \mathrm{kg}$, urinary sodium $83 \mathrm{mEq} / \mathrm{L}$, and urinary chloride $61 \mathrm{mEq} / \mathrm{L}$. Blood gas analysis showed mild compensated metabolic alkalosis ( $\mathrm{pH} 7.48, \mathrm{pCO}_{2} 44 \mathrm{~mm}$ $\mathrm{Hg}, \mathrm{pO}_{2} 79 \mathrm{~mm} \mathrm{Hg}$, and $\mathrm{HCO}_{3} 28 \mathrm{mmol} / \mathrm{L}$ ).

Initial evaluation showed a hypotonic hyponatremia with high urine osmolality and elevated urinary sodium concentration (superior to $40 \mathrm{mmol} / \mathrm{L}$ ). Clinically, the patient had no symptoms suggestive of orthostatic hypotension, no tachycardia, and was not apparently dehydrated. Conventional hypovolemia biochemical markers showed a normal hematocrit, mild elevated blood urea, low serum uric acid levels and a mild metabolic alkalosis. Thyroid function tests and serum cortisol levels were normal. At this point, we assumed CSW as the most probable diagnosis and started hypertonic saline with $1.5 \% \mathrm{NaCl} 2 \mathrm{~L} /$ day with oral water intake of $1 \mathrm{~L} /$ day. The patient showed a progressive improvement in sodium levels with normalization at day 4 (Fig. 1).

At this point, the urine osmolality was low and the patient excreted diluted urine. After suspension of intravenous fluids, we started the patient on salt tablets and the sodium levels remained normal. The patient was discharged to our outpatient clinic. At 3 months follow-up, the patient remained stable.

\section{Discussion}

CSW is a rare and probably underdiagnosed syndrome. Current knowledge suggests it might occur in patients without clinical evidence of cerebral disease, making the term RSW a more appropriate denomination [5]. Nonetheless, the term CSW remains the designation, although some authors think that the syndrome itself might not exist $[8,9]$. Most of the available epidemiological data derive from patients with aneurysmal subarachnoid hemorrhage (SHA) with a prevalence varying from $6.5 \%$ to $75 \%$. Other than SHA, this syndrome has also been described in patients with CNS infections, primary and metastatic CNS neoplasms and following neurosurgery [2].

SLE can affect the nervous system at multiple levels with neurological and psychiatric manifestations which may be secondary to vasculopathy or direct effect of antibodies [10-12]. Acute cerebrovascular disease is a known case of hyponatremia in the form of SIADH or CSW, but there is not a known association with chronic lesions [13]. There is no established association between dysnatremias and SLE other than hyponatremia associated with some pharmacological therapies $[14,15]$. In our patient, the SLE initial manifestation was a coma of unknown origin that after thorough investigation proved to be secondary to SLE. At that point, the patient already had a mild hyponatremia $(130 \mathrm{mEq} / \mathrm{L})$ interpreted as secondary to a pharmacological SIADH after cyclophosphamide pulses. The patient was discharged and advised to make water restriction which he fulfilled. The fact that, not only did the hyponatremia not resolve, but it aggravated made us firstly question if the patient was in fact carrying out the water restriction. After confirmation that he was liable, we began to think that the initial diagnosis could be incorrect so we admitted the patient to reevaluate the cause of the hyponatremia. As in most published recommendations, our initial approach in evaluating a patient with hyponatremia was to determine the serum and urinary osmolality [16-19]. Our patient had a hypoosmolar hyponatremia with high urinary osmolality which showed impaired water excretion (as the normal kidney response would be the excretion of maximum dilute urine). Then we measured the urine sodium concentration which was inappropriately elevated $(>40 \mathrm{mmol} / \mathrm{L})$. Since the patient was not taking diuretics nor had advanced kidney disease, after ex- 
Table 1. Characteristics of SIADH and CSW

\begin{tabular}{lll} 
& CSW & SIADH \\
\hline Plasma volume & $\downarrow$ & Normal or $\uparrow$ \\
Water balance & Negative & Normal or $\uparrow$ \\
Serum osmolality & $\downarrow$ & $\downarrow$ \\
Signs and symptom of dehydration & Present in most cases & Absent \\
Hematocrit & $\uparrow$ or normal & Unchanged \\
Plasma BUN/creatine & $\uparrow$ or normal & $\downarrow$ \\
Urine sodium & $\uparrow \uparrow$ & $\uparrow$ \\
Urine volume & $\uparrow \uparrow$ & $\downarrow$ or normal \\
Uric acid & $\downarrow$ & $\downarrow$ \\
FEUrate after correction of hyponatremia & $\uparrow$ & Normal \\
Treatment & Normal or hypertonic saline & Water restriction \\
& Fludrocortisone & Hypertonic saline and furosemide \\
\hline
\end{tabular}

BUN: blood urea nitrogen; FEUrate: excretional fraction of urate; CSW: cerebral salt wasting; SIADH: syndrome of inappropriate antidiuretic hormone secretion.

clusion of hypothyroidism and adrenal insufficiency the two remaining diagnoses were SIADH and CSW. Their differentiation was the major clinical challenge. Both are syndromes characterized by a hypoosmolar hyponatremia with high urine osmolality and elevated urinary sodium concentration (Table 1).

The only clinical difference is the state of the extracellular volume, being euvolemic or hypervolemic in SIADH and hypovolemic in RSW [1, 20-22]. Therefore, accurate determination of the patient's volume status is the key to differentiating these syndromes. The problem is that the determination of volume status is notoriously difficult to perform accurately in routine clinical practice. Conventional biochemical markers that normally reflect hypovolemia such as renin and aldosterone are unreliable (as in the setting of CSW they do not reflect the degree of hypovolemia as they are secondarily suppressed) and other more common laboratory data are not independently diagnostic and lack specificity. So in the absence of obvious clinical features of hypovolemia, markers like an elevation in serum bicarbonate, blood urea nitrogen concentration, hematocrit or elevated serum uric acid levels would indicate a hypovolemic state but only in patients with normal renal function $[2,20,21]$. The problems with our patient were some confounding factors: firstly the elevated blood urea could be secondary to the steroids he was taking, he had normal hematocrit and the serum uric acid level was low as it is expected in both disorders; the only clue was the mild metabolic alkalosis which could be interpreted as secondary to excessive renal fluid loss with secondary volume contraction. There is evidence that the urate clearance expressed by the fractional excretion of urate (FEUrate) can be of assistance in differentiating SIADH from CSW. Some authors have shown a possible FEUrate has been consistently increased to $>11 \%$ in SIADH and RSW and has a unique relationship to serum sodium in both disorders: in SIADH, correction of hyponatremia will normalize FEUrate to $4-11 \%$ as compared to being persistently increased to $>11 \%$ in RSW. It has actually been proposed to include FEUrate in the clinical algorithm of evaluation of the hyponatremic patient $[4,5,18,21]$. In our hospital, we were unable to determine FEUrate but after thorough clinical integration, and specially knowing that water restriction was not helping our patient, we considered CSW the most probable diagnosis and started appropriate treatment. The patient's favorable evolution proved our suspicion correct. The mainstay of therapy for CSW is replacement of the sodium and water. One approach is to initially administer normal saline to restore intravascular volume and test if the urine becomes diluted which is expected to occur in $24-48 \mathrm{~h}$ in CSW. If the urine becomes diluted, serum sodium should be carefully corrected to prevent osmotic demyelination. Fludrocortisone can be used to enhance sodium reabsorption [2, 23]. Other approach is to use a hypertonic saline $(1.5-3 \% \mathrm{NaCl})$ and if necessary add the mineralocorticoid and additional salt tablets $[1,2,24]$. In most cases, the CSW is resolved after the resolution of the acute insult (typically 5 - 7 days) but in our patient, this did not happen. The question is if it is possible for $\mathrm{CSW}$ to extend further in time. Is it possible that in patients with neurolupus the insult carries on the cascade of peptide production that perpetuates the hyponatremia? Or is there any other mechanism not already known? A lot of questions remain unanswered. Perhaps the knowledge that this disorder also occurs in patients with lupus can, in the future, elucidate the pathophysiological mechanisms of both diseases.

\section{Conclusions}

CSW is an established disorder characterized by a hypoosmolar hyponatremia with inappropriate RSW originally described in the setting of aneurysmal SAH. The growing knowledge in understanding this disease has permitted its diagnosis in other diseases with CNS involvement as SLE. However, new diagnostic approaches are essential for better and prompt diagnosis of this disorder in order to start appropriate treatment. 


\section{Grant Support}

None.

\section{Competing Interest}

The authors declare that they do not have any competing interest.

\section{References}

1. Cerda-Esteve M, Cuadrado-Godia E, Chillaron JJ, PontSunyer C, Cucurella G, Fernandez M, Goday A, et al. Cerebral salt wasting syndrome: review. Eur J Intern Med. 2008;19(4):249-254.

2. Yee AH, Burns JD, Wijdicks EF. Cerebral salt wasting: pathophysiology, diagnosis, and treatment. Neurosurg Clin N Am. 2010;21(2):339-352.

3. Betjes MG. Hyponatremia in acute brain disease: the cerebral salt wasting syndrome. Eur J Intern Med. 2002;13(1):9-14.

4. Maesaka JK, Imbriano L, Mattana J, Gallagher D, Bade N, Sharif S. Differentiating SIADH from Cerebral/Renal Salt Wasting: Failure of the Volume Approach and Need for a New Approach to Hyponatremia. J Clin Med. 2014;3(4):1373-1385.

5. Maesaka JK, Imbriano LJ, Ali NM, Ilamathi E. Is it cerebral or renal salt wasting? Kidney Int. 2009;76(9):934938.

6. Deslarzes T, Turini P, Friolet R, Meier P. [Cerbral salt wasting syndrome versus SIADH]. Rev Med Suisse. 2009;5(225):2281-2284.

7. Oh JY, Shin JI. Syndrome of inappropriate antidiuretic hormone secretion and cerebral/renal salt wasting syndrome: similarities and differences. Front Pediatr. 2014;2:146.

8. Singh S, Bohn D, Carlotti AP, Cusimano M, Rutka JT, Halperin ML. Cerebral salt wasting: truths, fallacies, theories, and challenges. Crit Care Med. 2002;30(11):25752579.

9. Momi J, Tang CM, Abcar AC, Kujubu DA, Sim JJ. Hyponatremia-what is cerebral salt wasting? Perm J.
2010;14(2):62-65.

10. Joseph FG, Lammie GA, Scolding NJ. CNS lupus: a study of 41 patients. Neurology. 2007;69(7):644-654.

11. Joseph FG, Scolding NJ. Neurolupus. Pract Neurol. 2010;10(1):4-15.

12. Muscal E, Brey RL. Neurologic manifestations of systemic lupus erythematosus in children and adults. Neurol Clin. 2010;28(1):61-73.

13. Kim DK, Joo KW. Hyponatremia in patients with neurologic disorders. Electrolyte Blood Press. 2009;7(2):5157.

14. Gilbar PJ, Richmond J, Wood J, Sullivan A. Syndrome of inappropriate antidiuretic hormone secretion induced by a single dose of oral cyclophosphamide. Ann Pharmacother. 2012;46(9):e23.

15. Jayachandran NV, Chandrasekhara PK, Thomas J, Agrawal S, Narsimulu G. Cyclophosphamide-associated complications: we need to be aware of SIADH and central pontine myelinolysis. Rheumatology (Oxford). 2009;48(1):89-90.

16. Maxwell AP. Diagnosis and management of hyponatraemia: AGREEing the guidelines. BMC Med. 2015;13:31.

17. Thompson C, Berl T, Tejedor A, Johannsson G. Differential diagnosis of hyponatraemia. Best Pract Res Clin Endocrinol Metab. 2012;26(Suppl 1):S7-15.

18. Milionis HJ, Liamis GL, Elisaf MS. The hyponatremic patient: a systematic approach to laboratory diagnosis. CMAJ. 2002;166(8):1056-1062.

19. Filippatos TD, Liamis G, Christopoulou F, Elisaf MS. Ten common pitfalls in the evaluation of patients with hyponatremia. Eur J Intern Med. 2016;29:22-25.

20. Harrigan MR. Cerebral salt wasting syndrome. Crit Care Clin. 2001;17(1):125-138.

21. Moritz ML. Syndrome of inappropriate antidiuresis and cerebral salt wasting syndrome: are they different and does it matter? Pediatr Nephrol. 2012;27(5):689-693.

22. Sterns RH, Silver SM. Cerebral salt wasting versus SIADH: what difference? J Am Soc Nephrol. 2008;19(2):194-196.

23. Lee P, Jones GR, Center JR. Successful treatment of adult cerebral salt wasting with fludrocortisone. Arch Intern Med. 2008;168(3):325-326.

24. Younas H, Sabir O, Baig I, Tarif N. Cerebral salt wasting: a report of three cases. J Coll Physicians Surg Pak. 2015;25(1):73-75. 